\title{
Isoliquiritigenin Inhibits Metastatic Breast Cancer Cell-induced Receptor Activator of Nuclear Factor Kappa-B Ligand/Osteoprotegerin Ratio in Human Osteoblastic Cells
}

\section{SHORT}

COMMUNICATION

\author{
Sun Kyoung Lee ${ }^{1,2}$, Kwang-Kyun Park ${ }^{1,2}$, Ki Rim Kim ${ }^{3}$, Hyun-Jeong Kim ${ }^{1,2}$, Won-Yoon Chung ${ }^{1,2}$ \\ 'Department of Oral Biology, Oral Cancer Research Institute, BK21 PLUS Project, Yonsei University College of Dentistry, ${ }^{2}$ Department of Applied \\ Life Science, Yonsei University Graduate School, Seoul, ${ }^{3}$ Department of Dental Hygiene, College of Science and Technology, Kyungpook National \\ University, Sangju, Korea
}

\begin{abstract}
Bone destruction induced by the metastasis of breast cancer cells is a frequent complication that is caused by the interaction between cancer cells and bone cells. Receptor activator of nuclear factor kappa-B ligand (RANKL) and the endogenous soluble RANKL inhibitor, osteoprotegerin (OPG), directly play critical roles in the differentiation, activity, and survival of osteoclasts. In patients with bone metastases, osteoclastic bone resorption promotes the majority of skeletal-related events and propagates bone metastases. Therefore, blocking osteoclast activity and differentiation via RANKL inhibition can be a promising therapeutic approach for cancer-associated bone diseases. We investigated the potential of isoliquiritigenin (ISL), which has anti-proliferative, anti-angiogenic, and anti-invasive effects, as a preventive and therapeutic agent for breast cancer cell-induced bone destruction. ISL at non-toxicity concentrations significantly inhibited the RANKL/OPG ratio by reducing the production of RANKL and restoring OPG production to control levels in hFOB1.19 cells stimulated with conditioned medium (CM) of MDA-MB-231 cells. In addition, ISL reduced the expression of cyclooxygenase-2 in hFOB1.19 cells stimulated by CM of MDA-MB-231 cells. Therefore, ISL may have inhibitory potential on breast cancer-induced bone destruction.
\end{abstract}

(J Cancer Prev 2015;20:281-286)

Key Words: Isoliquiritigenin, Breast cancer, Bone metastasis, RANK ligand, Osteoblastic cells

\section{INTRODUCTION}

Bone metastasis from breast cancer leads to severe bone destruction through the interaction between cancer cells and bone cells. ${ }^{1}$ Osteolytic bone destruction significantly decreases the survival rate of patients with metastatic breast cancer. ${ }^{2}$ Receptor activator of nuclear factor kappa-B ligand (RANKL) and osteoprotegerin (OPG) are key molecules in bone-resorbing osteoclast differentiation and activation. RANKL from osteoblasts binds to receptor activator of nuclear factor kappa-B (RANK) on the surface of preosteoclasts and promotes the maturation of osteoclasts. OPG secreted by osteoblasts obstructs the binding of RANKL and RANK. Whereas a balance between RANKL and OPG is maintained in normal physiology, various cytokines or chemokines secreted by breast cancer cells disrupt the balance between RANKL and $\mathrm{OPG}^{3}$ Consequently, the number of bone-resorbing osteoclasts and bone destruction are abnormally increased in patients with metastatic breast cancer. ${ }^{4}$ Therefore, RANKL can be a strategic therapeutic target for patients with metastatic breast cancer. ${ }^{5}$

Isoliquiritigenin (ISL) (Fig. 1A) is a flavonoid derived from licorice and shallot. ${ }^{6}$ ISL has diverse pharmacological activities such as anti-inflammatory, anti-diabetic, anti-angiogenic, and anti-osteoclastic properties. ${ }^{7-10}$ In addition, ISL has strong anticancer effects in various types of cancers. ${ }^{11-13}$ In breast cancer, ISL inhibits the metastasis of human breast cancer cells through

Received November 25, 2015, Revised December 9, 2015. Accepted December 9, 2015

Correspondence to: Won-Yoon Chung

Department of Oral Biology, Yonsei University College of Dentistry, 50-1 Yonsei-ro, Seodaemun-gu, Seoul 03722, Korea

Tel: +82-2-2228-3057, Fax: +82-2-364-7113, E-mail: wychung@yuhs.ac, ORCID: Won-Yoon Chung, http://orcid.org/0000-0001-8428-9005

Copyright (C) 2015 Korean Society of Cancer Prevention

(c) This is an Open Access article distributed under the terms of the Creative Commons Attribution Non-Commercial License (http://creativecommons.org/licenses/by-nc/4.0) which permits unrestricted non-commercial use, distribution, and reproduction in any medium, provided the original work is properly cited. 
<smiles>O=C(/C=C/c1ccc(O)cc1)c1ccc(O)cc1O</smiles>

B

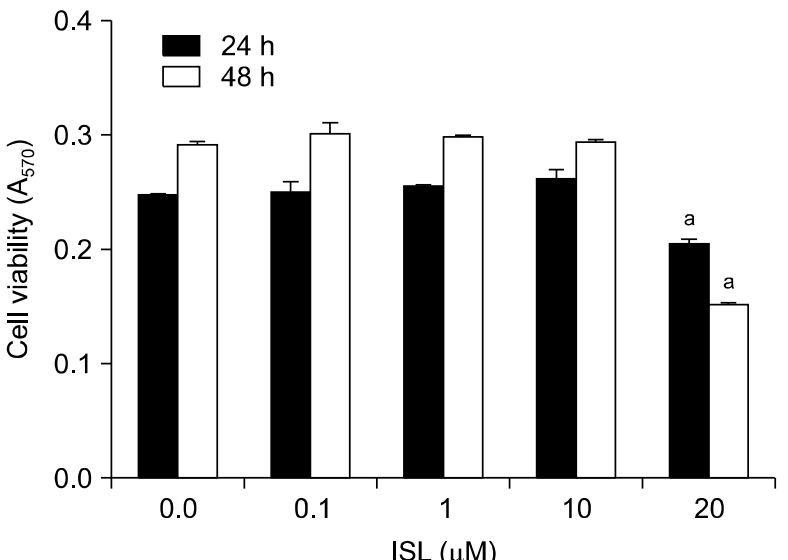

C
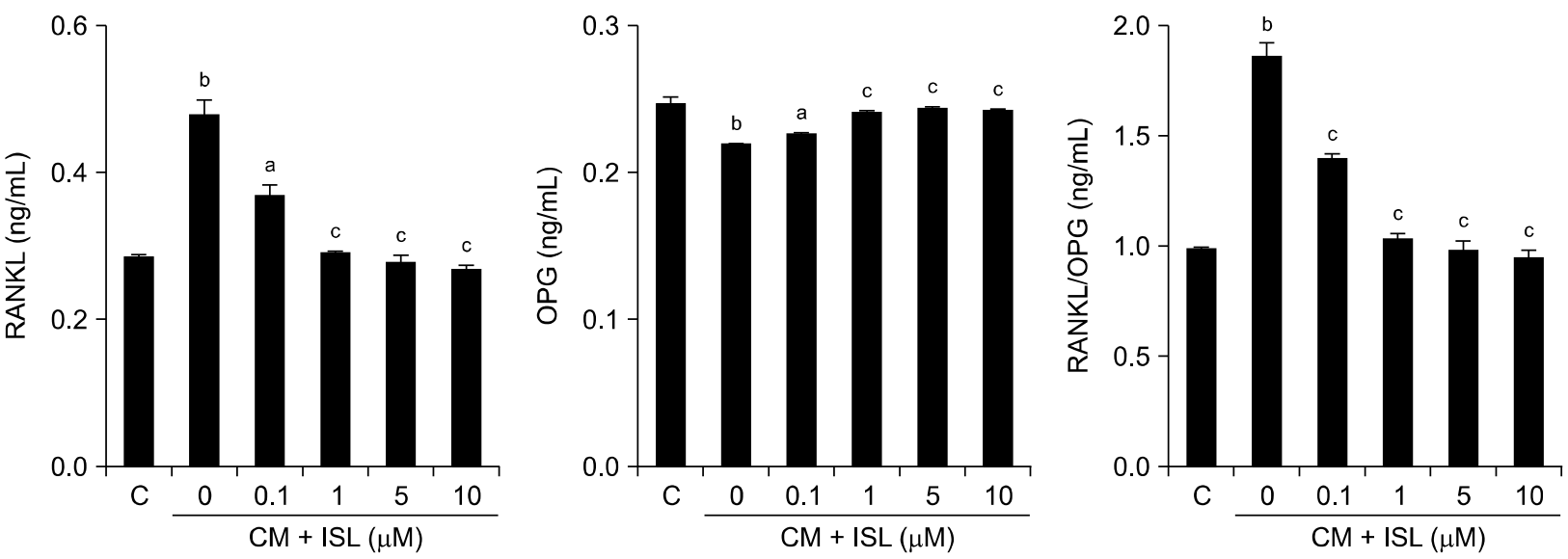

D
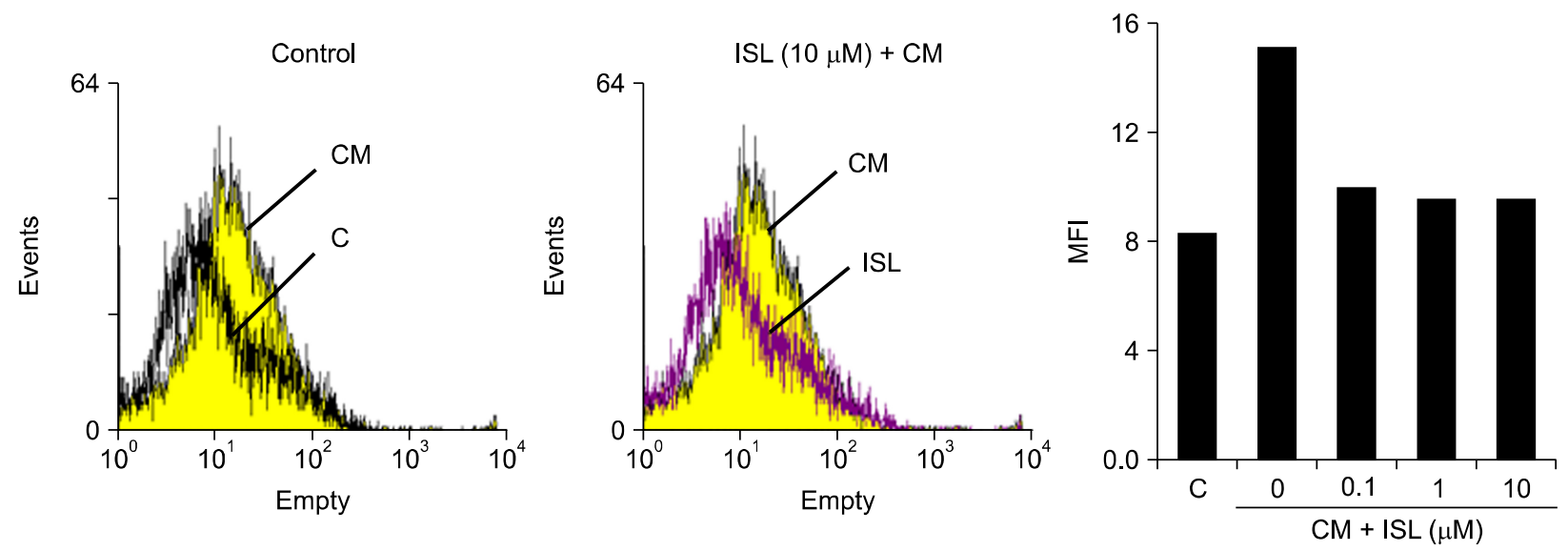

Figure 1. The effect of isoliquiritigenin (ISL) on the receptor activator of nuclear factor kappa-B ligand (RANKL)/osteoprotegerin (OPG) ratio in hFOB1.19 cells treated with conditioned medium (CM) of MDA-MB-231 cells. (A) Chemical structure of ISL. (B) hFOB1.19 cells were cultured with indicated concentrations of ISL for 24 hours and 48 hours. The cell viability was analyzed by the MTT assay. Data are expressed as the means $\pm \mathrm{SE},{ }^{\mathrm{a}} P<0.01$ vs. untreated cells. (C) hFOB1.19 cells were treated with CM and ISL at noncytotoxic concentrations for 24 hours. RANKL and OPG levels in the culture media were determined using commercially available ELISA kits. Data are expressed as the means $\pm \mathrm{SE},{ }^{\mathrm{b}} p<0.01$ vs. control; ${ }^{\mathrm{a}} P<0.05,{ }^{\mathrm{c}} P<0.01 \mathrm{vs}$. hFOB1.19 cells treated with CM of MDA-MB-231 cells. (D) hFOB1.19 cells were incubated with CM of MDA-MB-231 cells and ISL $(1-10 \mu \mathrm{M})$ for 48 hours. Then, the cells were harvested and analyzed by fluorescence activated cell sorter (FACS) as described in Materials and Methods. MFI, mean fluorescence intensity; C, control. 
preventing anoikis resistance, as well as the migration and invasion of BT-549 and MDA-MB-231 cells. ${ }^{14}$ ISL also induces growth inhibition and apoptosis through downregulation of the arachidonic acid metabolic network and the deactivation of PI3K/Akt in MCF-7 and MDA-MB-231 cells. ${ }^{15}$

In the present study to verify the potential of ISL as a promising agent for control of breast cancer bone metastasis, we investigated whether ISL could affect RANKL and OPG expression in osteoblastic cells stimulated with conditioned medium (CM) of metastatic breast cancer cells.

\section{MATERIALS AND METHODS}

\section{Materials}

ISL, vitamin $C$ (ascorbic acid), vitamin $\mathrm{D}_{3}$, vitamin $\mathrm{K}_{3}$, MTT, dimethylsulfoxide, and bovine serum albumin (BSA) were purchased from Sigma (St. Louis, MO, USA). Leibovitz's L-15 (L-15) medium, Dulbecco's Modified Eagle's Medium:Nutrient Mixture F-12 (Ham) (1:1) (DMEM/F12), FBS, and antibiotics were purchased from Gibco BRL (Grand Island, NY, USA). Monoclonal antibody (mAb) against human RANKL was obtained from R\&D Systems (Minneapolis, MN, USA), and polyclonal anti-human COX-2 antibody was purchased from Cayman Chemical (Ann Arbor, MI, USA). Normal goat immunoglobulin G (IgG) and normal rabbit IgG, as well as an enhanced chemiluminescence (ECL) kit were obtained from Santa Cruz Biotechnology (Dallas, TX, USA).

\section{Cell culture}

MDA-MB-231 human breast cancer cells and hFOB1.19 human fetal osteoblast cells were obtained from American Type Culture Collection (Manassas, VA, USA). MDA-MB-231 cells were cultured in L-15 supplemented with $10 \%$ FBS and $1 \%$ penicillin/ streptomycin. hFOB1.19 cells were maintained DMEM/F12 with $10 \% \mathrm{FBS}$ and $1 \%$ penicillin/streptomycin, and, at $80 \%$ to $90 \%$ confluence, cultured in differentiation medium for an additional 2 to 3 days prior to treatment with CM of breast cancer cells and/or ISL. Differentiation medium consisted of DMEM:F-12 (1:1), 50 $\mu \mathrm{g} / \mathrm{mL}$, vitamin $\mathrm{C}, 10^{-8} \mathrm{M}$ vitamin $\mathrm{D}_{3}, 10^{-8} \mathrm{M}$ vitamin $\mathrm{K}_{3}$, and $10 \%$ charcoal-stripped FBS to minimize exposure to hormones and growth factors. ${ }^{16}$

\section{MTT assay}

hFOB1.19 osteoblasts were seeded into 96-well culture plates at a density of $1 \times 10^{4}$ cells/well. Twenty-four hours later, hFOB1.19 cells were cultured for 24 hours and 48 hours in respective serum-free media with various concentrations of ISL.
Cell viability was measured using an MTT assay. The absorbance was determined at $570 \mathrm{~nm}$ using a microplate reader (Bio-Rad Laboratories, Hercules, CA, USA).

\section{Preparation of conditioned medium derived from MDA-MB-231 cells}

MDA-MB-231 cells $\left(1 \times 10^{6}\right.$ cells $)$ were plated in T75 culture flasks with 10 mL DMEM:F-12 (1:1) supplemented with 10\% FBS, and incubated at $37^{\circ} \mathrm{C}$. When the cells were nearly confluent, the medium was changed to serum-free DMEM:F-12 (1:1). After 24 hours incubation, the medium was collected as $\mathrm{CM}$, aliquoted, and frozen at $-20^{\circ} \mathrm{C}$. For the following experiments, $70 \% \mathrm{CM}$ in differentiation medium was used. ${ }^{16}$

\section{ELISA}

hFOB1.19 cells were cultured in 96-well plates $\left(1 \times 10^{5}\right.$ cells/well) in DMEM/F12 containing 10\% FBS for 24 hours and then treated with 70\% CM of MDA-MB-231 cells and ISL (0.1-10 $\mu \mathrm{M})$. After 24 hours incubation, cultured medium of the hFOB1.19 cells was collected. The RANKL and OPG protein levels in the collected medium were quantified using commercially available ELISA kits (EIAab, Guangguguoji, China) according to the manufacturer's protocols.

\section{Flow cytometric (fluorescence activated cell sorter) analysis}

hFOB1.19 cells $\left(1 \times 10^{6}\right.$ cells $)$ were treated with $70 \% \mathrm{CM}$ and ISL at the indicated concentrations for 48 hours. The cells were detached with $2 \mathrm{mM}$ EDTA and subsequently incubated in phosphate-buffered saline (PBS) with $1 \%$ BSA on ice for 30 minutes. Aliquots $\left(2 \times 10^{5}\right.$ cells $)$ were incubated with $100 \mu \mathrm{L}$ RANKL mAb $(10 \mu \mathrm{g} / \mathrm{mL})$ on ice for 1 hour. The cells were washed twice with $\mathrm{Mg}^{2+}$ - and $\mathrm{Ca}^{2+}$-free PBS with $1 \% \mathrm{BSA}$ and resuspended in $\mathrm{Mg}^{2+}$ - and $\mathrm{Ca}^{2+}$-free PBS containing $1 \% \mathrm{BSA}$ and a 1:50 dilution of fluorescein isothiocyanate-conjugated IgG. After incubation with secondary antibody for 1 hour at $4^{\circ} \mathrm{C}$, the cells were washed again. Ten thousand events were stored as list mode data for further analysis using WinMDI software (Windows Multiple Document Interface Flow Cytometry Application, 1993-1998 Joseph Trotter). ${ }^{16}$

\section{Reverse transcription-PCR}

hFOB1.19 cells $\left(1 \times 10^{6}\right.$ cells) were treated with 70\% CM and ISL for 6 hours. Total RNA from hFOB1.19 cells was extracted using the TRIzol ${ }^{\mathbb{R}}$ Reagent (Invitrogen, Carlsbad, CA, USA). Single stranded cDNA was transcribed from the RNA ( $2 \mu \mathrm{g})$ using 
Promega's reverse transcription system (Madison, WI, USA). PCR was carried out in a reaction mixture containing cDNA $(2 \mu \mathrm{g})$, $\mathrm{MgCl}_{2}$ (25 mM), dNTPs (10 mM), primers (1 pmol), and Taq polymerase (1 unit) (Takara, Shiga, Japan) with the following primers: COX-1, forward 5'-CATCCTCGACGGCATCTCAGC-3'; reverse 5'-TTGGGTCAGGGGTGGTTATTG-3'; COX-2, forward 5' -ATGACTTCCAAGCTGGCCGT-3'; reverse 5'-CCTCTTCAAAAACT TCTCCACACC-3'; GAPDH, forward 5'-GTCAGTGGTGGACCTGAC CT-3'; reverse 5'-AGGGGTCTACATGGCAACTG-3'. The amplification consisted of 30 cycles with an annealing temperature of $63^{\circ} \mathrm{C}$ for COX-1, $57.5^{\circ} \mathrm{C}$ for COX-2 and $52^{\circ} \mathrm{C}$ for GAPDH. The PCR products were electrophoresed, and the detected bands were analyzed with the TINA program ver. 2.10e (Raytest, Straubenhardt, Germany).

\section{Western blotting}

hFOB1.19 cells $\left(1 \times 10^{6}\right.$ cells $)$ were cultured in media containing $70 \% \mathrm{CM}$ and/or ISL at the indicated concentration for 6 hours, and were lysed with the radioimmunoprecipitation assay buffer. The protein concentration was measured with a bicinchoninic acid (BCA) kit (Pierce, Rockford, IL, USA). Equal amounts of protein $(40 \mu \mathrm{g})$ were loaded onto a gel for SDS-PAGE. The blots were transferred to a polyvinylidene difluoride membrane (Millipore, Billerica, MA, USA). The membrane was blocked with $5 \%$ skim milk in Tris-buffered saline with Tween 20 (TBST) and then incubated with the primary antibodies for COX-2 $(1: 1,000)$ and $\beta$-actin $(1: 2,000)$ in 3\% skim milk in TBST for 24 hours at $4^{\circ} \mathrm{C}$. After washing, the blots were incubated for 1 hour with secondary antibody coupled to horseradish peroxidase $(1: 2,000)$ and visualized with the ECL kit.

\section{Statistical analysis}

Statistical analysis was performed with SPSS statistical software ver. 21 (IBM, Endicott, NY, USA). Data are expressed as mean \pm SE. Data were analyzed by means of the Student's t-test to express differences between the two groups. Results with values of $P<0.05$ and $P<0.01$ were considered statistically significant.

\section{RESULTS}

1. Isoliquiritigenin inhibited the receptor activator of nuclear factor kappa-B ligand/osteoprotegerin ratio in osteoblastic cells stimulated with conditioned medium of MDA-MB-231 breast cancer cells

We first examined the cytotoxicity of ISL on hFOB1.19 human osteoblastic cells. When hFOB1.19 cells were exposed to various concentrations of ISL, the viability of hFOB1.19 cells was suppressed at $20 \mu \mathrm{M}$ by $17 \%$ with 24 hours treatment and by $47 \%$ with 48 hours treatment (Fig. 1B). Next, we analyzed the secreted levels of soluble RANKL and OPG in hFOB1.19 cells treated with CM of MDA-MB-231 cells and ISL at noncytotoxic concentrations, using ELISA kits. ISL treatment significantly inhibited the secreted levels of RANKL and rescued those of OPG into the culture medium of osteoblastic cells stimulated with $\mathrm{CM}$ of MDA-MB-231 cells. Consequently, the elevated RANKL/OPG ratio by CM of MDA-MB-231 cells was inhibited by ISL treatment in a dose-dependent manner (Fig. 1C). In addition, we detected the expression level of membrane-bound RANKL in hFOB1.19 cells using fluorescence activated cell sorter analysis. CM of breast cancer cells increased the membrane-bound RANKL expression, but ISL reduced the CM-induced level of membrane-bound RANKL by $37 \%$ at $10 \mu \mathrm{M}$ (Fig. 1D).

2. Isoliquiritigenin inhibited the expression level of COX-2 in conditioned medium-stimulated osteoblastic cells

COX-2 is one of the well-known molecules that regulate the expression of RANKL. ${ }^{17,18}$ Thus, we further examined the effect of ISL on the expression of COX-2 in CM-treated hFOB1.19 cells. Reverse transcription (RT)-PCR data showed that CM of MDAMB-231 cells increased the mRNA expression of COX-2, but ISL at non-cytotoxic concentrations blocked its CM-induced mRNA expression in hFOB1.19 osteoblastic cells. COX-1 mRNA expression was not changed significantly by either ISL or CM of MDA-MB-231 cells in hFOB1.19 cells (Fig. 2A). Western blot analysis also indicated that ISL significantly inhibited the protein levels of COX-2 in hFOB1.19 cells exposed to CM of MDA-MB-231 cells (Fig. 2B).

\section{DISCUSSION}

Bone metastasis is frequently observed in patients with advanced breast cancer, and the mortality rate is significantly higher in patients with bone metastasis than in patients without bone metastasis. ${ }^{2}$ Metastatic breast cancer cells metastasize to bone and secrete various osteolytic factors. ${ }^{1}$ These factors stimulate osteoblasts that are important regulators of bone metabolism and abnormally increase the expression of RANKL, which is a key promoting factor for osteoclast differentiation and activation. ${ }^{19}$ Consequently, excessive bone resorption by osteoclasts is rapidly increased in patients with metastatic breast 
A
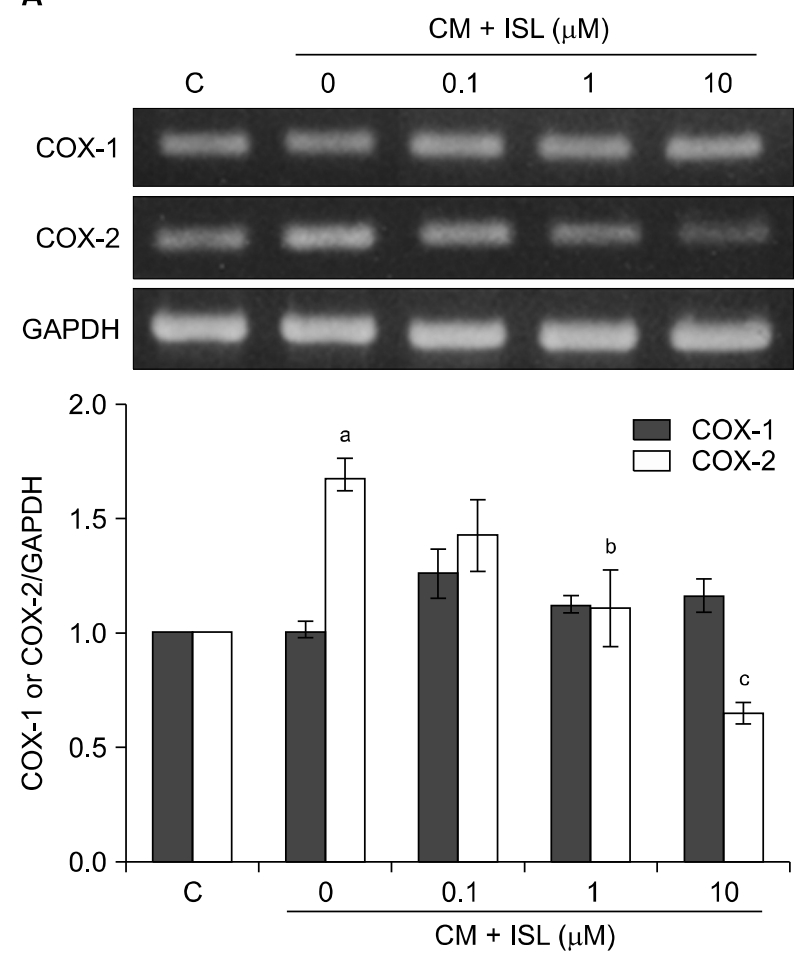

B
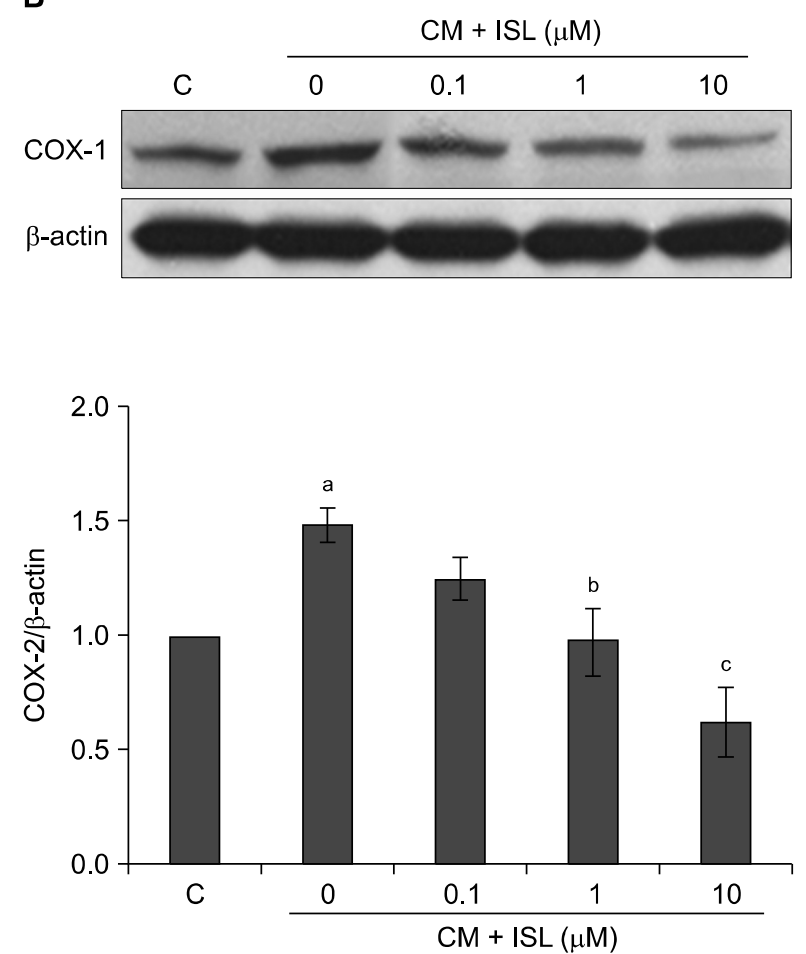

Figure 2. The effect of isoliquiritigenin (ISL) on mRNA and protein expressions of COX-2 in hFOB1.19 cells stimulated by conditioned medium (CM) of MDA-MB-231 cells. (A) hFOB1.19 cells were incubated with CM of MDA-MB-231 cells and ISL (1-10 $\mu$ M) for 1 hour. The mRNA expression of COX-1 and COX-2 was analyzed by reverse transcription-PCR. (B) hFOB1.19 cells were incubated with CM of MDA-MB-231 cells and ISL $(1-10 \mu \mathrm{M})$ for 6 hours. Proteins were extracted and subjected to western blotting using the specific antibody against COX-2. Data are expressed as the means $\pm \mathrm{SE},{ }^{\mathrm{a}} P<0.01$ vs. control; ${ }^{\mathrm{b}} P<0.05,{ }^{\mathrm{c}} P<0.01 \mathrm{vs}$. hFOB1.19 cells treated with CM of MDA-MB-231 cells. GAPTH, glyceraldehyde-3-phosphate dehydrogenase; C, control.

cancer. ${ }^{4}$ Several growth factors released from the bone matrix by osteoclast-mediated bone resorption contribute to the proliferation and survival of cancer cells. These amplified interactions between breast cancer cells and bone cells, referred to as a 'vicious cycle', make the condition difficult to treat. ${ }^{20,21}$ Therefore, controlling osteoblastic RANKL expression in the bone microenvironment with breast cancer metastases can be a promising strategy for the prevention and treatment of cancer-associated bone loss.

ISL has potent anti-inflammatory, anti-angiogenic, and anticancer effects. ${ }^{8,10-13}$ In particular, ISL prevents the metastatic potential of breast cancer cells through inhibition of matrix metalloproteinase activities and the PI3K/Akt signaling pathway. ${ }^{14,15}$ In our previous study, we found that ISL inhibited RANKL-induced osteoclastogenesis. ${ }^{16}$ The RANKL and OPG from osteoblasts are important molecules in the differentiation of osteoclasts. $^{22}$ Thus, we further found that ISL at non-cytotoxic concentrations inhibited the RANKL/OPG ratio by blocking the expression of soluble and membrane-bound RANKL and the decrease in OPG production in human osteoblastic cells stimulated with CM of metastatic breast cancer cells.

COXs are the enzymes that mediate the conversion of arachidonic acid to prostaglandins (PGs). ${ }^{23}$ Whereas COX-1 is expressed constitutively in most tissues and plays an important role in the maintenance of homeostasis, COX-2 is an inducible enzyme contributing to the elevated production of PGs in inflammation and cancer. ${ }^{24}$ Recent studies have demonstrated that COX-2 expression regulates the production of PGE2, which is one of the osteolytic factors involved in RANKL expression in osteoblasts. ${ }^{17}$ Mice lacking COX-2 showed reduced bone resorption in response to parathyroid hormone. ${ }^{18}$ PGE2 produced by cancer cells or osteoblasts binds to the osteoblastic EP4 receptor, which is one of the PGE receptor subtypes, and induces RANKL expression to stimulate bone resorption. ${ }^{25}$ Additionally, COX-2 has been known to be involved in breast cancer bone metastasis. ${ }^{26}$ In our study, ISL downregulated the expression levels of COX-2 mRNA and protein elevated by CM of MDA-MB-231 cells in hFOB1.19 cells.

Taken together, ISL inhibited the RANKL/OPG ratio and COX-2 expression in human osteoblast hFOB1.19 cells stimulated with 
CM of metastatic breast cancer MDA-MB-231 cells. Thus, ISL can be a beneficial agent to inhibit and treat breast cancer cellassociated bone diseases by blocking the interaction between cancer cells and bone cells via the inhibition of osteoblastic RANKL expression.

\section{ACKNOWLEDGMENTS}

This work was carried out with the support of "Cooperative Research Program for Agriculture Science \& Technology Development (Project No. PJ011578)" Rural Development Administration, Republic of Korea.

\section{CONFLICTS OF INTEREST}

No potential conflicts of interest were disclosed.

\section{REFERENCES}

1. Mundy GR. Metastasis to bone: causes, consequences and therapeutic opportunities. Nat Rev Cancer 2002;2:584-93.

2. Theriault RL, Theriault RL. Biology of bone metastases. Cancer Control 2012;19:92-101.

3. Patel LR, Camacho DF, Shiozawa Y, Pienta KJ, Taichman RS. Mechanisms of cancer cell metastasis to the bone: a multistep process. Future Oncol 2011;7:1285-97.

4. Suva LJ, Washam C, Nicholas RW, Griffin RJ. Bone metastasis: mechanisms and therapeutic opportunities. Nat Rev Endocrinol 2011;7:208-18.

5. Dougall WC, Holen I, González Suárez E. Targeting RANKL in metastasis. Bonekey Rep 2014:3:519.

6. Peng F, Du Q, Peng C, Wang N, Tang H, Xie X, et al. A review: the pharmacology of isoliquiritigenin. Phytother Res 2015;29:969-77.

7. Gaur R, Yadav KS, Verma RK, Yadav NP, Bhakuni RS. In vivo anti-diabetic activity of derivatives of isoliquiritigenin and liquiritigenin. Phytomedicine 2014:21:415-22.

8. Kang SW, Choi JS, Choi YJ, Bae JY, Li J, Kim DS, et al. Licorice isoliquiritigenin dampens angiogenic activity via inhibition of MAPK-responsive signaling pathways leading to induction of matrix metalloproteinases. J Nutr Biochem 2010;21:55-65.

9. Zhu L, Wei H, Wu Y, Yang S, Xiao L, Zhang J, et al. Licorice isoliquiritigenin suppresses RANKL-induced osteoclastogenesis in vitro and prevents inflammatory bone loss in vivo. Int J Biochem Cell Biol 2012;44:1139-52

10. Kumar S, Sharma A, Madan B, Singhal V, Ghosh B. Isoliquiritigenin inhibits IkappaB kinase activity and ROS generation to block TNF-alpha induced expression of cell adhesion molecules on human endothelial cells. Biochem Pharmacol 2007;73:1602-12.
11. Park I, Park KK, Park JH, Chung WY. Isoliquiritigenin induces G2 and $\mathrm{M}$ phase arrest by inducing DNA damage and by inhibiting the metaphase/anaphase transition. Cancer Lett 2009;277:174-81.

12. Kwon GT, Cho HJ, Chung WY, Park KK, Moon A, Park JH. Isoliquiritigenin inhibits migration and invasion of prostate cancer cells: possible mediation by decreased JNK/AP-1 signaling. J Nutr Biochem 2009;20:663-76.

13. Chen X, Wu Y, Jiang Y, Zhou Y, Wang Y, Yao Y, et al. Isoliquiritigenin inhibits the growth of multiple myeloma via blocking IL-6 signaling. J Mol Med (Berl) 2012;90:1311-9.

14. Zheng H, Li Y, Wang Y, Zhao H, Zhang J, Chai H, et al. Downregulation of COX-2 and CYP 4A signaling by isoliquiritigenin inhibits human breast cancer metastasis through preventing anoikis resistance, migration and invasion. Toxicol Appl Pharmacol 2014:280:10-20.

15. Li Y, Zhao H, Wang Y, Zheng H, Yu W, Chai H, et al. Isoliquiritigenin induces growth inhibition and apoptosis through downregulating arachidonic acid metabolic network and the deactivation of PI3K/Akt in human breast cancer. Toxicol Appl Pharmacol 2013; 272:37-48.

16. Lee SK, Park KK, Park JH, Lim SS, Chung WY. The inhibitory effect of roasted licorice extract on human metastatic breast cancer cell-induced bone destruction. Phytother Res 2013;27:1776-83.

17. Liu XH, Kirschenbaum A, Yao S, Levine AC. Interactive effect of interleukin-6 and prostaglandin E2 on osteoclastogenesis via the OPG/RANKL/RANK system. Ann N Y Acad Sci 2006;1068:225-33.

18. Okada Y, Lorenzo JA, Freeman AM, Tomita M, Morham SG, Raisz LG, et al. Prostaglandin G/H synthase-2 is required for maximal formation of osteoclast-like cells in culture. J Clin Invest 2000; 105:823-32.

19. Roodman GD. Bone-breaking cancer treatment. Nat Med 2007; 13:25-6.

20. Hiraga T, Myoui A, Hashimoto N, Sasaki A, Hata K, Morita Y, et al. Bone-derived IGF mediates crosstalk between bone and breast cancer cells in bony metastases. Cancer Res 2012;72:4238-49.

21. Guise TA, Chirgwin JM. Transforming growth factor-beta in osteolytic breast cancer bone metastases. Clin Orthop Relat Res 2003;(415 Suppl):S32-8.

22. Boyle WJ, Simonet WS, Lacey DL. Osteoclast differentiation and activation. Nature 2003;423:337-42.

23. Smith WL, DeWitt DL, Garavito RM. Cyclooxygenases: structural, cellular, and molecular biology. Annu Rev Biochem 2000;69:14582.

24. Zha S, Yegnasubramanian V, Nelson WG, Isaacs WB, De Marzo AM. Cyclooxygenases in cancer: progress and perspective. Cancer Lett 2004:215:1-20.

25. Miyaura C, Inada M, Suzawa T, Sugimoto Y, Ushikubi F, Ichikawa A, et al. Impaired bone resorption to prostaglandin E2 in prostaglandin E receptor EP4-knockout mice. J Biol Chem 2000;275: 19819-23.

26. Singh B, Berry JA, Shoher A, Ayers GD, Wei C, Lucci A. COX-2 involvement in breast cancer metastasis to bone. Oncogene 2007; 26:3789-96. 